\title{
Investigation of the possibility for reducing agglomeration of aerosol nanoparticles by using the needle-plate corona charger
}

\author{
A. A. Efimov, P. V. Arsenov, V. V. Ivanov \\ Moscow Institute of Physics and Technology, Russia, 141700 Dolgoprudny, Institutskiy per., 9 \\ newaldan@gmail.com
}

\begin{abstract}
The results of the research are given to show the possibility of reducing the agglomeration of aerosol nanoparticles using the needle-plate corona charger. It has been found that the charger fulfills the functions of an electrofilter-separator, precipitating large particles of agglomerates at a size more than $250 \mathrm{~nm}$, leaving smaller non-agglomerated particles in the flow. Using the developed charger allows us to significantly reduce the agglomeration of particles at sufficiently low aerosol flow rates. As a result of changing the parameters of the charger (corona discharge current from 35 to $215 \mu \mathrm{A}$, aerosol flow through the charger from 33 to $250 \mathrm{l} / \mathrm{min}$ and the corona polarity), the mean particle size decreases more than in 1.5 -fold.
\end{abstract}

Keywords: the needle-plate charger, agglomeration, aerosol nanoparticles, aerosol, the multi-spark discharge generator.

Received: 27 November 2017

\section{Introduction}

Recent years have seen an increase in the use of aerosol nano-particles to create new materials and devices [1]. It is known that the size of aerosol nanoparticles affects the characteristics of the materials and devices in which they are used. In this context, controlling the size of aerosol nanoparticles is a significant task. It is also a known fact that the size of the aerosol nanoparticles increases as a result of the agglomeration process, to be more precise as a result of the collision of particles during their movement in the gas stream.

In this regard, researchers are developing various methods to reduce the agglomeration of nanoparticles. The most effective and simple method for reducing the agglomeration of nanoparticles is diluting the aerosol flow with clean gas [2]. However, this method is not always applicable in practice because there are physical limitations to increasing the dilution ratio of the aerosol.

Charging nanoparticles is an alternative method for reducing the agglomeration process. Therefore, the corona discharge has been one of the most common methods for charging aerosol nanoparticles. There are many works devoted to the study of charging the aerosol nanoparticles in the corona discharge [3,4]. However, most of them have a disadvantage related to the complexity of the design and the high cost of chargers, which limits their various applications. Bearing all this in mind, in this work a relatively simple and cheap unipolar needle-plate charger was designed and investigated to reduce the agglomeration of aerosol nanoparticles. In particular, in this paper, we investigated the effect of the parameters of the charger (the value and polarity of the corona discharge current and gas flow) on the agglomeration of nanoparticles, namely on the mean particle size and the particle size distribution.

\section{Experiment}

In the experiments we used aerosol nanoparticles of $\mathrm{Al}_{2} \mathrm{O}_{3}$, which were obtained by a multi-spark discharge generator (m-SDG), as a result of electric erosion of the electrode material from $\mathrm{Al}$ in air [5,6], see Fig. 1. Then the received nanoparticles were sent to a charger (NPC). The charger was an asymmetric needle-plate discharge gap located in a dielectric tube with an internal diameter of $45 \mathrm{~mm}$. A steel needle with a radius of curvature of about $40 \mu \mathrm{m}$ was used as the corona electrode and was located at a distance of $10 \mathrm{~mm}$ from the opposite electrode, which was made in the form of a plate of $100 \mathrm{~mm}$ long and $30 \mathrm{~mm}$ wide. When a high voltage was applied between the needle and the plate, a region of high electric field intensity appeared at the tip of the needle, resulting in ionization of the gas molecules and ignition of the corona discharge. As a high-voltage source, the VIDN-30 source was used, and the corona discharge current was measured with an Agilent U1253B multimeter.

Aerosol nanoparticles entering the corona discharge region acquired a unipolar charge due to the adsorption of ions or electrons on their surfaces, depending on the polarity of the corona electrode. As a result of the collection of unipolar charge their further agglomeration was prevented due to Coulomb repulsion [7]. 


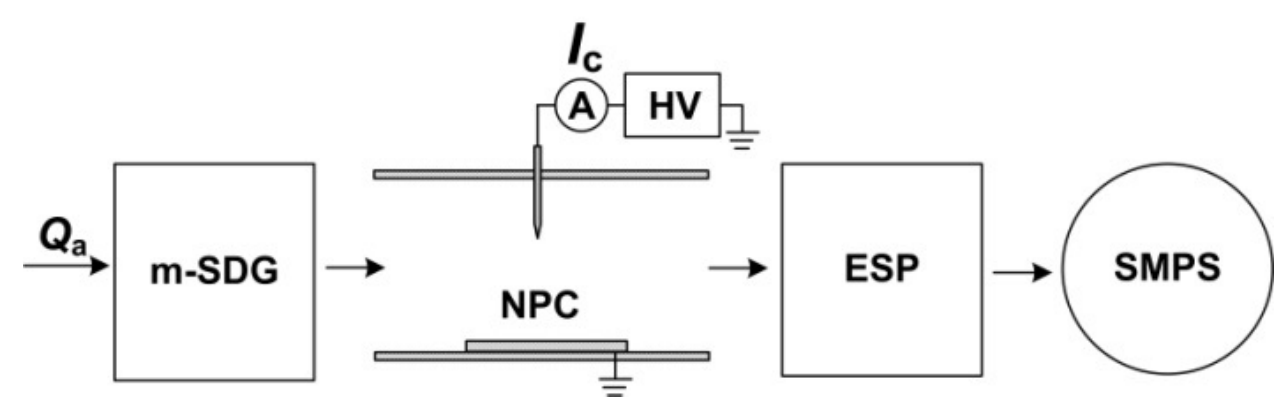

FIG. 1. Conditional scheme of the experiment to study the possibilities of reducing the agglomeration of aerosol nanoparticles using a needle-plate charger. The following abbreviations are used: m-SDG - multi-spark discharge generator, NPC - needle-plate charger, ESP - electrostatic precipitator, SMPS - aerosol spectrometer

At the output of the charger an electrostatic precipitator (ESP) was placed, where all charged particles were captured in order to determine the fraction of charged particles in the aerosol. The capture efficiency of aerosol nanoparticles $E_{e f f}$ in the charger was defined by the following formula:

$$
E_{\text {eff }}=\left(1-\frac{n_{\text {out }}}{n_{\text {in }}}\right) \cdot 100 \%,
$$

where $n_{i n}$ is the number concentration of particles in the initial aerosol, $n_{\text {out }}$ is the number concentration of particles after passing through the charger.

Using the aerosol spectrometer (SMPS) model TSI 3936, the particle size distribution was measured and from its analysis the degree of agglomeration of the particles was determined. The agglomeration of particles was investigated by varying the basic parameters of the charger. Specifically, we researched the influence of corona discharge current from 35 to $215 \mu \mathrm{A}$, charging polarity (negative and positive) and aerosol flow rate through the charger from 33 to $250 \mathrm{l} / \mathrm{min}$.

\section{Results and discussion}

When studying the influence of the corona discharge current on the agglomeration, it was found that an increase in current from 35 to $215 \mu \mathrm{A}$ promotes a decrease in the agglomeration of nanoparticles, namely a decrease in their mean particle size $d_{m}$ from $117 \pm 8$ to $87 \pm 6 \mathrm{~nm}$, respectively (Fig. 2a). Along with this, there is also a decrease in the width of the particle size distribution, namely the geometric standard deviation (GSD) is reduced from $1.61 \pm 0.01$ to $1.58 \pm 0.01$, respectively. It should be noted that the initial aerosol without charge had a broader particle size distribution of GSD $=1.65 \pm 0.01$ and the mean particle size $158 \pm 11 \mathrm{~nm}$. The obtained result can be explained as follows: in accordance with the theory of charging, it is known that large particles are charged more efficiently and acquire a larger number of charges than the small ones [8]. Therefore, it follows that larger particles in the electric field will have a higher electrical mobility $Z_{p}$ in accordance with the following formula:

$$
Z_{p}=\frac{n_{p} e C}{3 \pi \mu d_{p}}
$$

where $n_{p}$ is the charge per particle, $e$ is the elementary unit of charge, $\mu$ is the viscosity of the gas, $d_{p}$ is the particle diameter, $C$ is the Cunningham correction.

As a result, in the corona discharge field, large agglomerates will precipitate more efficiently while small agglomerates will leave the charger without depositing on the electrodes. This explanation is confirmed by the graph in Fig. 2b, which shows the dependence of the capture efficiency of the charged particles in an electric field on the particle size for different values of the corona discharge current.

In Fig. 2b, we can see that most efficiently captured particles are of the submicron range with dimensions of more than $250 \mathrm{~nm}$, the filtration efficiency of which approaches $80 \%$. However, for particles with sizes less than $250 \mathrm{~nm}$, a noticeable decrease in the efficiency of their capture is observed.

A qualitative model that clearly demonstrates the process of reducing agglomeration of particles after passing through the charger is shown in Fig. 3. The model is constructed using experimental data for corona discharge current $I_{c}=35 \mu \mathrm{A}$, negative polarity and aerosol flow rate $Q_{a}=33 \mathrm{l} / \mathrm{min}$ through the charger.

This model shows the deposition and charging of particles of three size fractions - small (15\% of all particles), medium ( $70 \%$ of all particles) and large (15\% of all particles). For each of the particle fractions, the part of 

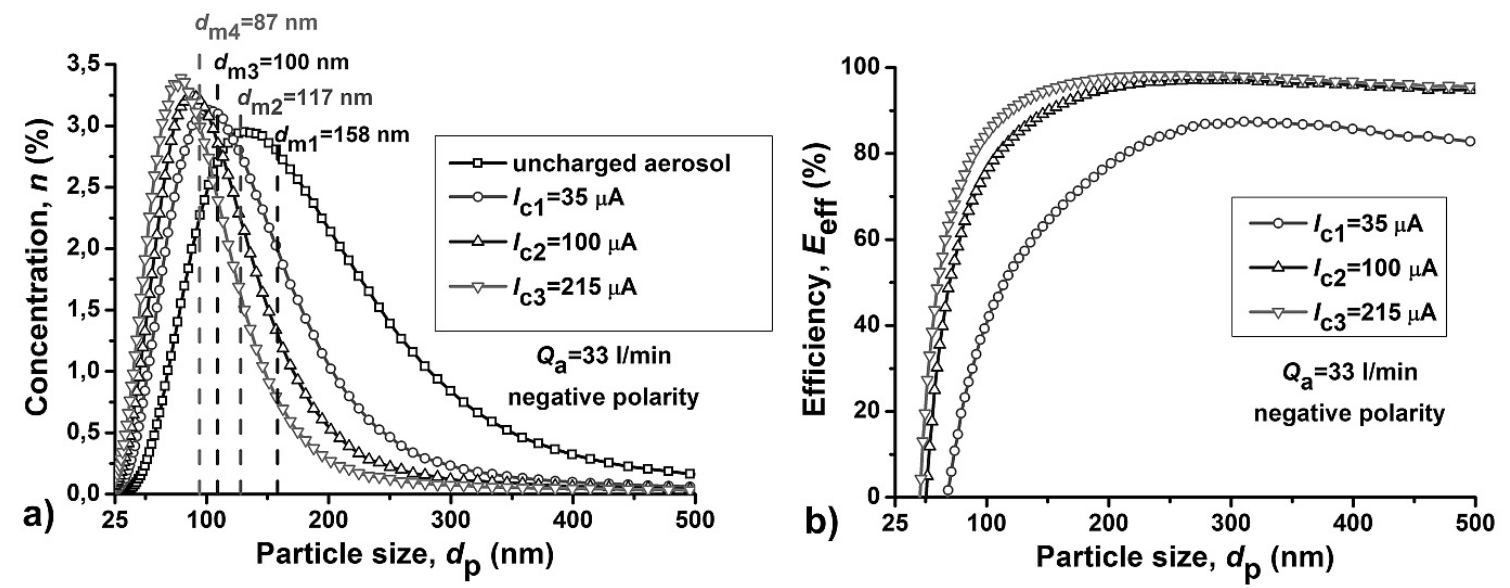

FIG. 2. Influence of the corona discharge current $I_{c}$ in the needle-plate charger on (a) the particle size distribution and (b) the particle capture efficiency in the charger

charged particles $E_{c h a r}$ and the capture efficiency $E_{\text {eff }}$ in the charger are determined by formulas (3, 4 and 1), respectively. The proportion of charged particles in the initial aerosol and after passing through the charger $E_{\text {char }}^{\text {out }}$ :

$$
\begin{aligned}
& E_{\text {char }}^{\text {out }}=\left(1-\frac{n_{\text {esp }}}{n_{\text {out }}}\right) \cdot 100 \%, \\
& E_{\text {char }}^{\text {in }}=\left(1-\frac{n_{\text {esp }}}{n_{\text {in }}}\right) \cdot 100 \%,
\end{aligned}
$$

where $n_{e s p}$ is the number concentration of neutral particles measured at the output of the electrostatic precipitator.

In Fig. 3, charged particles are shaded in gray. From Fig. 3, it can be seen that at the output of the charger there are more medium (63\% of all particles) and small particles (33\% of all particles) than larger particles (4\% of all particles). This redistribution of the proportions of small, medium and large particles in comparison with the initial aerosol was mainly due to the precipitation of the large particles. Fig. 3 shows that the fraction of charged particles after passing of the aerosol through the charger increases from $45 \pm 3 \%$ to $67 \pm 4 \%$ in comparison with the initial aerosol, which also contributes to the reduction of agglomeration as a result of Coulomb repulsion.

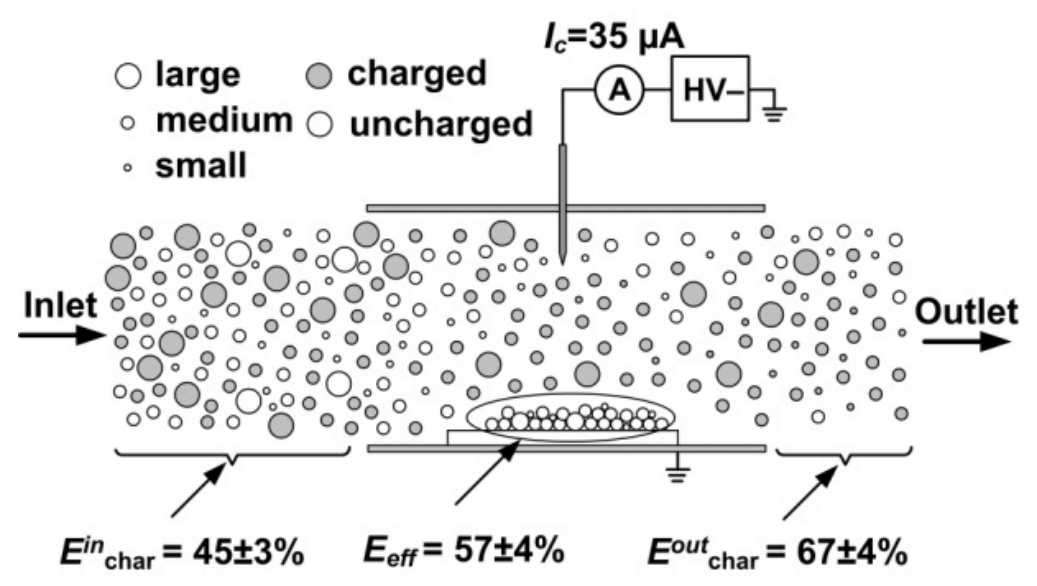

FIG. 3. Qualitative model of the experiment demonstrating the process of reducing the agglomeration of particles after passing through the needle-plate charger

While investigating the influence of corona discharge polarity at a current of $35 \mu \mathrm{A}$, it was established that negative polarity shows a greater effect on reducing the agglomeration of nanoparticles compared with the positive one (Fig. 4). In particular, when using a negative corona discharge, the mean particle size $d_{m}$ is 0.7 times smaller than with a positive corona discharge, $135 \pm 10$ and $100 \pm 8 \mathrm{~nm}$, respectively, while the original uncharged aerosol had a mean particle size of $161 \pm 11 \mathrm{~nm}$ (Fig. 4a). 

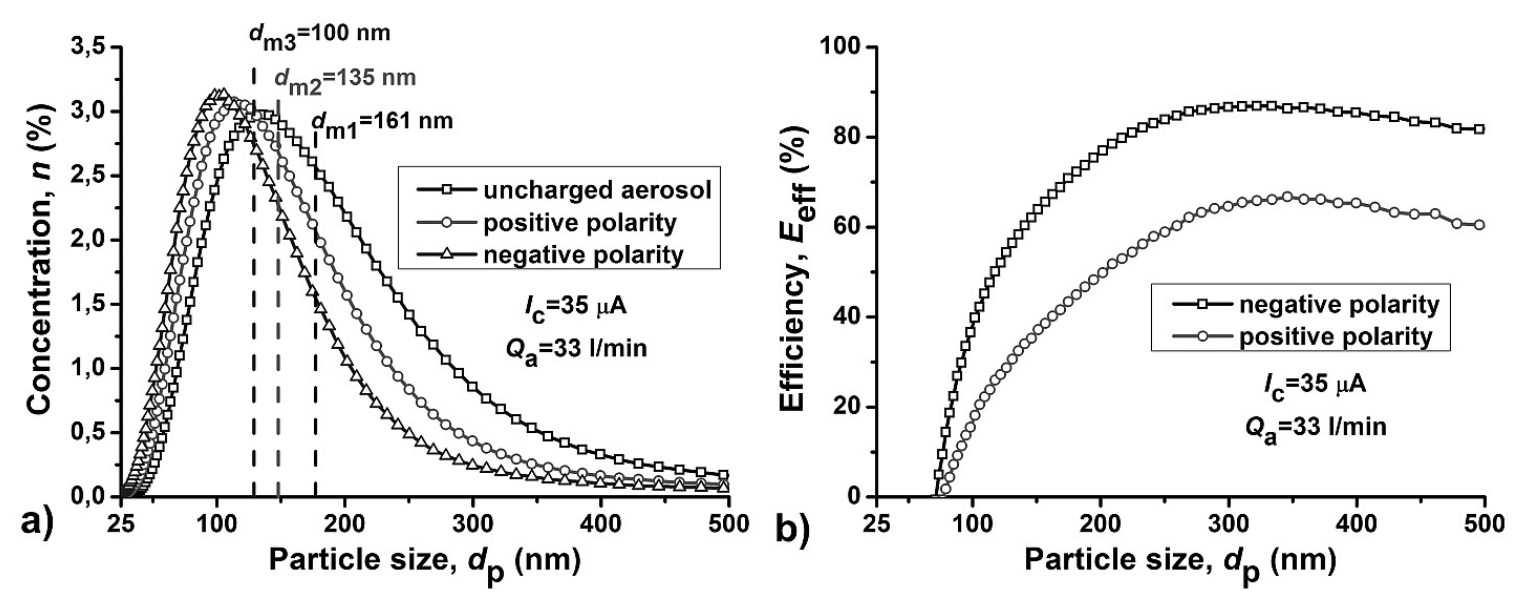

FIG. 4. The influence of polarity of corona discharge in the charger on (a) particle size distribution and (b) capture efficiency

A more effective reduction in the agglomeration of particles with the use of a negative corona discharge is explained by the fact that a higher efficiency of charging aerosol nanoparticles is achieved due to the higher electrical mobility of electrons in comparison with the mobility of positive ions, $\sim 4 \cdot 10^{2} \mathrm{~cm}^{2} / \mathrm{V} \cdot \mathrm{s}$ and $\sim 2 \mathrm{~cm} / \mathrm{V} \cdot \mathrm{s}$, respectively. This assumption is qualitatively confirmed in Fig. 4b, which shows that the capture efficiency of particle with negative charging is higher than with positive charging over the whole range of sizes from 75 to $500 \mathrm{~nm}$.

The influence of the aerosol flow through the charger on its agglomeration was also investigated. Fig. 5 shows the particle size distributions throughout the charger depending on the aerosol flow rate.
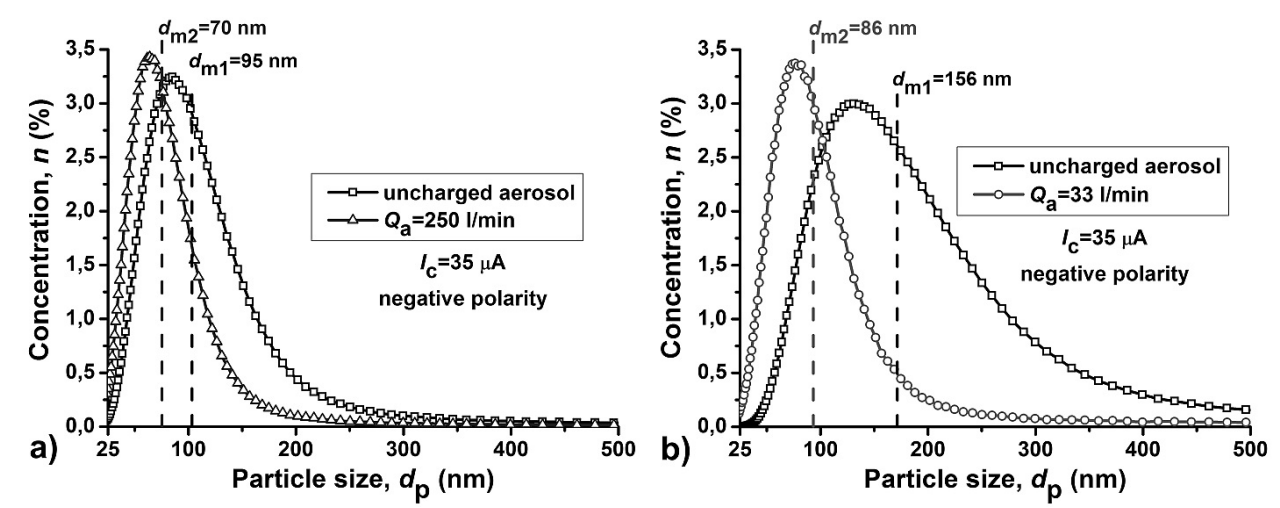

FIG. 5. Particle size distribution of uncharged and charged aerosol in the conditions of the aerosol flow rate at (a) $250 \mathrm{l} / \mathrm{min}$ and (b) $33 \mathrm{l} / \mathrm{min}$

In Fig. 5 we can see that at the aerosol flow rate of $2501 / \mathrm{min}$ (Fig. 5a), the mean particle size $d_{m}$ of the initial aerosol is less than at the aerosol flow rate of $33 \mathrm{l} / \mathrm{min}$ (Fig. 5b), $95 \pm 8$ and $156 \pm 14 \mathrm{~nm}$, respectively. This is mainly due to a decrease in the numerical concentration of aerosol particles as a result of their dilution with a clean gas stream. It should also be noted that when the aerosol passes through the charger, there is an additional reduction in the agglomeration of particles, which manifests itself in a decrease in their mean particle size $d_{m}$ from $95 \pm 8$ to $70 \pm 7 \mathrm{~nm}$ and from $156 \pm 14$ to $86 \pm 7 \mathrm{~nm}$, at $250 \mathrm{l} / \mathrm{min}$ and $33 \mathrm{l} / \mathrm{min}$, respectively. It is worth mentioning that with lower gas flow rate through the charger, more efficient charging of particles is achieved, and as a consequence, the mean particle size changes drastically. Thus, the use of a charger can significantly reduce the agglomeration of particles at fairly low aerosol flow rate.

\section{Conclusions}

According to the results of the experiments, it has been established that the use of a corona needle-plate charger leads to a significant reduction in the agglomeration of particles: that is in a number of performed experiments, it was possible to reduce the mean particle size $d_{m}$ more than in 1.5 -fold. 
It was found that the charger fulfills the functions of an electrofilter-separator, precipitating large particles of agglomerates at sizes more than $250 \mathrm{~nm}$, leaving smaller non-agglomerated particles in the flow.

It was determined that the efficiency of large agglomerate precipitation from the aerosol flow increased with an increase in corona discharge current $I_{c}$ from 35 to $215 \mu \mathrm{A}$, with changing corona current from a positive to a negative polarity and a decrease in the aerosol flow rate $Q_{a}$ from 250 to $33 \mathrm{l} / \mathrm{min}$ through the charger.

In the framework of this study, a model of the process of precipitation of large agglomerates is presented and the possibility of reducing agglomeration of particles at relatively low aerosol flow rate is shown.

\section{Acknowledgement}

This work in the experimental part of this study was supported by the Russian Science Foundation (project No. 15-19-00190) and in the theoretical part was supported by the grant of the President of Russian Federation for young scientists MK-2302.2017.8.

\section{References}

[1] Kortshagen U.R., Sankaran R.M., Pereira R.N., et al. Nonthermal plasma synthesis of nanocrystals: Fundamental principles, materials, and applications. Chem. Rev., 2016, 116(18), P. 11061-11127.

[2] Fissan H., Ristig S., Kaminski H., Asbach C., Epple M. Comparison of different characterization methods for nanoparticle dispersions before and after aerosolization. Anal. Methods, 2014, 6(18), P. 7324-7334.

[3] Intra P. An Overview of Unipolar Charger Developments for Nanoparticle Charging. Aerosol Air Qual. Res., 2011.

[4] M. Alonso Gámez, A. Hernández Sierra, F.J. Alguacil. Electrical charging of aerosol nanoparticles and sonne practical applications. Cargado eléctrico de partículas de aerosol en régimen cinético y algunas aplicaciones prácticas, 2003.

[5] Efimov A.A., Ivanov V.V., Bagazeev A.V., et al. Generation of aerosol nanoparticles by the multi-spark discharge generator. Tech. Phys. Lett., 2013, 39(12), P. 1053-1056.

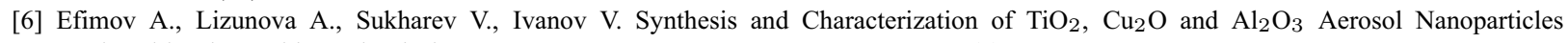
Produced by the Multi-Spark Discharge Generator. Korean J. Mater. Res., 2016, 26(3), P. 123-129.

[7] Qi C., Asbach C., Shin W.G., Fissan H., Pui D.Y.H. The Effect of Particle Pre-Existing Charge on Unipolar Charging and Its Implication on Electrical Aerosol Measurements. Aerosol Sci. Technol., 2009, 43(3), P. 232-240.

[8] Pfafflin J.R., Ziegler E.N. Encyclopedia of Environmental Science and Engineering: A-L. CRC Press, 2006. 